\title{
Comunicación Basada en Radio Cognitiva sobre Radio Definido por Software
}

\section{Communication Based in Cognitive Radio over Software Defined Radio}

\author{
Andy Vega \\ Universidad Nacional de Loja \\ Loja, Ecuador \\ andy.vega@unl.edu.ec \\ Orcid: 0000-0003-0106-6880
}

\author{
Andrea Guamo \\ Universidad Nacional de Loja \\ Loja, Ecuador \\ akguamom@unl.edu.ec
}

\begin{abstract}
Resumen - En la era de la tecnología inalámbrica, la escasez del espectro radioeléctrico plantea un grave dilema para los proveedores de servicios y los operadores de telecomunicaciones. La tecnología de Radio Cognitiva (RC) proporciona una solución innovadora para mejorar significativamente la utilización del espectro. El presente documento desarrolla y dispone la información necesaria para implementar un enlace de comunicación basado en Radio Cognitiva. Para realizar dicha tarea se utiliza herramientas como: software GNU Radio, en donde se implementa el sistema de comunicación que incluye el detector del espectro seleccionado y se pone en manifiesto la característica principal de la tecnología RC que debe ser consciente del entorno radioeléctrico con fines de optimizarlo y, a base del mismo, permite tomar decisiones de transmisión que no interrumpe en ningún momento la comunicación del enlace ni obstaculiza o perjudica la comunicación de un Usuario primario o licenciado. Además, se emplea herramientas de Radio definido por Software (SDR) que realizan tareas de radiocomunicación como procesamiento de banda base y las diferentes configuraciones necesarias para la radiocomunicación en la banda de Ultra Alta frecuencia (UHF), logrando así establecer un enlace de comunicación basado en Radio Cognitiva.
\end{abstract}

Palabras Clave: Radio, Cognitiva, Radio Definido por Software, GNU Radio, Sensado del Espectro, OFDM

\begin{abstract}
In this era of wireless technology, the scarcity of radio spectrum poses a serious dilemma for service providers and telecom operators. Cognitive Radio (CR) technology provides an innovative solution to significantly improve spectrum utilization. This document develops and provides the necessary information to implement a communication link based on Cognitive Radio. Tools such as GNU Radio software were used to carry out this task with the implementation of the communication system that contained the selected spectrum detector. It also revealed the main characteristic of the RC technology, which is, to be aware of the radio electric environment, to optimize it and, based on it, make transmission decisions that do not interrupt the communication of the link at any time or hinder or harm the communication of a primary or licensed User. Likewise, Software Defined Radio (SDR) tools were used. They perform radio communication tasks such as baseband processing and the different necessary settings for radio communication in the Ultra High Frequency (UHF) band, thus establishing a link of communication based on Cognitive Radio.
\end{abstract}

Keywords: Cognitive, Radio, Software Defined Radio, GNU Radio, Spectrum Sensing, OFDM

Sumario: I Introducción, II Radio Cognitiva, III Enlace de Comunicación basado en Radio Cognitiva, IV Resultados, V Discusión, VI Conclusiones.

Como citar: Vega, Andy., \& Guamo, Andrea. (2020). Comunicación Basada en Radio Cognitiva sobre Radio Definido por Software. Revista Tecnológica - Espol, 32(2). Recuperado a partir de http://www.rte.espol.edu.ec/index.php/tecnologica/article/view/736 


\section{INTRODUCCIÓN}

Las comunicaciones inalámbricas se han convertido en una parte indispensable de la vida diaria con demandas cada vez más altas. A consecuencia de la designación de forma estática del recurso natural no renovable, en este caso el espectro radioeléctrico, su empleo se ha realizado de manera poco eficiente e ineficaz. La proliferación de dispositivos y servicios inalámbricos para diferentes usos tales como las comunicaciones móviles, la seguridad pública, Wi-Fi y televisión sirven como ejemplo indiscutible de cuánto la sociedad moderna se ha vuelto dependiente del manejo del espectro radioeléctrico.

Recientes estudios del espectro radioeléctrico realizados por la Comisión Federal de Comunicaciones (FCC) -por sus siglas en inglés- muestra que la utilización del espectro en la banda de $0-6 \mathrm{GHz}$ varía de $15 \%$ al $85 \%[1]$, demostrando así que gran parte del espectro licenciado asignado está infrautilizado. La Radio Cognitiva nace como la tecnología que plantea una gestión del recurso natural de carácter limitado de manera mucho más eficiente y propone un acceso dinámico al espectro radioeléctrico. De esta forma, presenta un sistema de radiofrecuencia capaz de modificar sus parámetros de transmisión basándose en la interacción con el medio en el que opera y trabaja, con la premisa fundamental de la no interferencia con los usuarios licenciados o Usuarios Primarios[2][3]. Uno de los mecanismos más importantes del concepto cognitivo es la capacidad de medir, detectar, aprender y conocer los parámetros relacionados con las características del canal de radio, la disponibilidad de espectro y potencia, el entorno operativo de la radio, los requisitos y aplicaciones del usuario, las redes disponibles y nodos, políticas locales y otras restricciones operativas.

En años anteriores, los dispositivos de comunicación como el sistema de radio tradicional admitían un número fijo de canales y múltiples frecuencias, pero el problema residía en que el canal y la frecuencia deben elegirse a la hora del diseño y no en una etapa posterior porque no existe mecanismo para realizar ningún ajuste posterior al diseño. En indagación de una mejor solución a la problemática, se introduce un concepto denominado Software Defined Radio (SDR) -por sus siglas en inglés-,[4] el cual ejecuta la mayor parte de las funciones de un equipo de radio, inclusive las más importantes, por medio del software implementado en un ordenador.

Aunque la definición de SDR no es relativamente nueva, la mayor limitante que presenta es su falta de inteligencia para la toma de decisiones, con el fin de superar dicha restricción participa activamente RC, logra eliminar los inconvenientes de SDR. A continuación, en este apartado se cree fundamental exponer diversos trabajos relacionados a este tema, como un exordio importante al trabajo realizado.

De acuerdo con, [5] SDR ilustran un papel fundamental en la puesta en marcha de los sistemas de comunicación, el mismo enseña el diseño, desarrollo y validación de un sistema de detección de radio cognitivo basado en SDR con un nivel muy alto.

Mejía Ardila, Andrés Fernando [6] presentan un análisis ocupacional en la banda GSM para redes de RC, indicando la aplicación de la tecnología en una banda específica y los resultados obtenidos del mismo. Varios estudios coinciden en que la detección de espectro ha sido definida como la labor de obtener un sentido de espectro disponible, determinando la existencia de usuarios licenciados dentro de una región geográfica definida [7]. Algunos trabajos han centrado su investigación en encontrar un método de detección de espectro idóneo y eficaz, además de indicar las diferencias entre los mismos como ocurre en [8]. Detectar oportunamente el espectro es una capacidad importante de los sistemas de RC, los White Spaces presentan una oportunidad potencial para la comunicación inalámbrica.

Zhe Huang, Weidong Wang, Yinghai Zhang [9] proponen y plantean un estudio y posterior ejecución de Radio Cognitiva utilizando USRP, dicho análisis proporciona un punto de partida para la presente investigación. En [10] observamos cómo se realiza la tarea de sensado del espectro por medio de USRP, y usan dos diferentes detectores de espectro: el detector de energía y detector de ciclo-estacionariedad; y cómo por medio de la curva ROC verifican la veracidad de los algoritmos implementados y ejecutados.

El propósito de este artículo es proporcionar la base teórica y práctica para la implementación de un enlace de comunicaciones basado en Radio Cognitiva tomando como referencia el estándar IEEE 802.22. Se realiza el diseño y posterior ejecución en la herramienta de software GNU Radio junto con las herramientas de software SDR, en los Laboratorios de Antenas y Telecomunicaciones de la Universidad Nacional de Loja.

\section{A. Generalidades}

\section{RADIO COGNITIVA}

La Radio Cognitiva se basa en su uso potencial para el acceso dinámico al espectro: Radios que encuentran y usan "White Spaces". Por lo tanto, una radio cognitiva puede definirse como una radio inteligente, la cual puede programarse a base de su interacción con el entorno y configuración dinámica para alterar su comportamiento. De esta forma, lograr las condiciones óptimas de transmisión

Para que un dispositivo de RC pueda obtener todas las características antes mencionadas, en su tesis doctoral Mitola [11], declara que es necesario un ciclo cognitivo que realice una determinada función que conlleva a una compleja radio autoconsciente. En la Fig. 1 se observa los niveles del ciclo cognitivo.

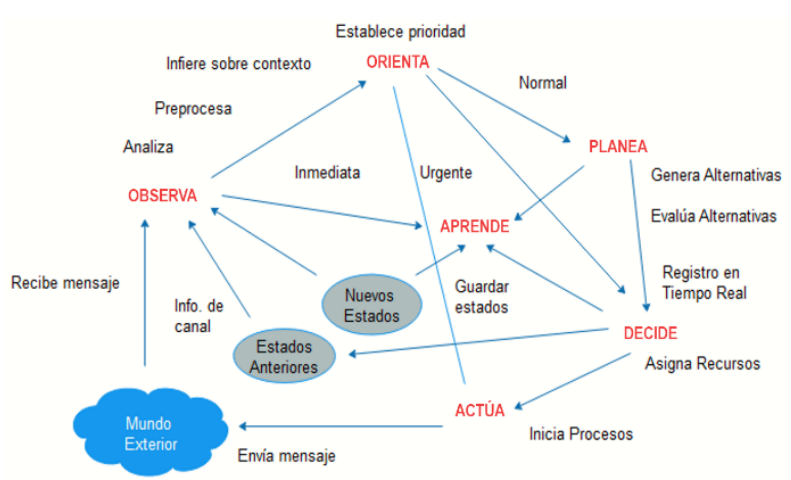

Fig. 1 Ciclo Cognitivo según Mitola 
En la terminología de radio cognitiva, el usuario primario (PU) -por sus siglas en inglés- se puede definir como el usuario que posee mayor prioridad en el empleo de cualquier parte específica del espectro. El usuario secundario (SU) -por sus siglas en inglés- es el que tiene una prioridad mucho menor. El usuario secundario obtiene acceso a este espectro de tal manera que no causa ningún tipo de interferencia al usuario principal ya existente. Por lo tanto, un usuario secundario debe tener capacidades de radio cognitiva, como detectar el espectro de manera confiable para verificar si la banda del espectro está siendo utilizada por algún usuario primario y cambiar sus propios parámetros de radio para explotar la banda no utilizada del espectro. La Fig. 2 ilustra lo anteriormente descrito.

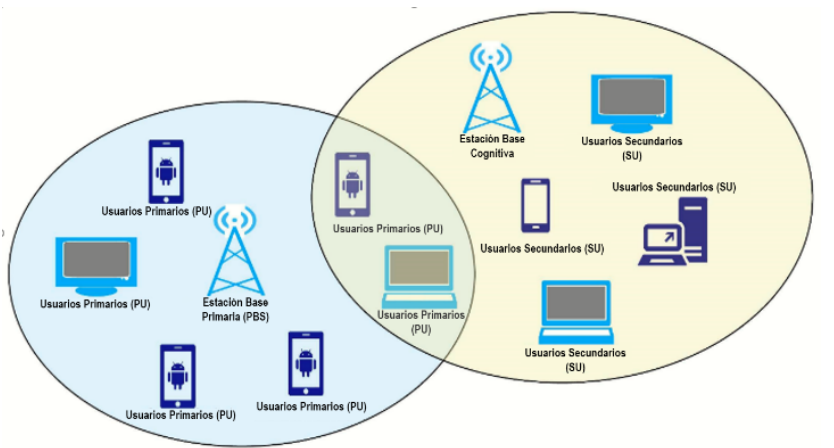

Fig. 2 Usuarios: Primarios y Secundarios en un Red CR

\section{B. Detección del Espectro}

Las técnicas de Detección de Espectro o Sensado, se convierte en el mecanismo esencial y crucial que permite a la RC detectar oportunidades de transmisión. Para minimizar la interferencia causada por los Usuarios Primarios, la tarea de detección de espectro realizada por un $\mathrm{RC}$ debe ser confiable y rápida.

Hasta el momento, se han propuesto varios enfoques para la detección del espectro, como el filtro combinado, la detección de energía (ED), la detección de características y la detección de wavelets [12]. En dichos métodos, ED utiliza la energía de la señal recibida en la SU para determinar la presencia de señales de la PU.

Este método simple puede recopilar información de ocupación del espectro de forma rápida. Su principal característica es que no requiere información previa sobre el comportamiento (modulación) de la señal recibida o del convertidor de señal analógica a digital (ADC) acciones obligatorias para los detectores de filtro acoplado y de ciclo estación respectivamente. Además, el estándar IEEE 802.22 incluye la detección de especies a través de ED en RC. Por lo tanto, un detector de energía es una opción óptima cuando la información sobre el canal no está disponible, y es el seleccionado para la implementación del presente trabajo.

Con el algoritmo de detección seleccionado, se presentan dos probabilidades de interés; probabilidad de detección $\mathrm{P}_{\mathrm{d}}$ y probabilidad de falsa alarma $\mathrm{P}_{\mathrm{fa}}$.

\section{Detector de Energía}

La disyuntiva del sensado de espectro puede ser modelado como un test de hipótesis, lo cual es equivalente a decidir entre 2 hipótesis: [13]

$$
y(t) \begin{cases}H_{0}=n(t) & \text { Señal Ausente } \\ H_{1}=s(t)+n(t) & \text { Señal Presente }\end{cases}
$$

Donde, $y(t)$ es la señal recibida por el usuario $\mathrm{CR}, \mathrm{H}_{1}$ indica que hay una señal presente, $\mathrm{H}_{0}$ indica ausencia de señal, $s(t)$ es señal transmitida por el $P U, n(t)$ es el ruido blanco Gaussiano indica aditivo de media nula (sus siglas en inglés AWGN).

El estadístico utilizado generalmente por este tipo de detectores es la energía media del total de las muestras observadas: [13]

$$
\mathrm{T}=\frac{1}{\mathrm{~N}} \sum_{\mathrm{t}=1}^{\mathrm{N}}|\mathrm{y}(\mathrm{t})|^{2} \gtrless \lambda
$$

Donde $\mathrm{T}$ es el tamaño del vector de observación. El funcionamiento de cualquier detector debe ser descrito mediante $\mathbf{P}_{\mathbf{f a}}$, y $\mathbf{P}_{\mathbf{d}}$. Una falsa alarma se da cada vez que $T<\lambda$ en el caso de que $\boldsymbol{H}_{\mathbf{0}}$ sea cierta, mientras que la detección ocurre cuando $T>\lambda$ siendo $\boldsymbol{H}_{\mathbf{1}}$ cierta, Las ecuaciones 3-5 muestra lo anteriormente descrito en forma matemática.

$$
\begin{gathered}
P_{f a}=P_{r}\left(T>\lambda \mid H_{0}\right)=Q\left(\frac{\lambda-\left(\sigma_{s}^{2}-\sigma_{n}^{2}\right)}{\left(\sigma_{s}^{2}-\sigma_{n}^{2}\right) \sqrt{\frac{2}{N}}}\right) \\
P_{d}=P_{r}\left(T>\lambda \mid H_{1}\right)=Q\left(\frac{\lambda-\sigma_{n}^{2}}{\sigma_{n}^{2} \sqrt{\frac{2}{N}}}\right)
\end{gathered}
$$

Donde función Q es: [14]

$$
Q(x)=\frac{1}{2 \pi}\left(\int_{x}^{\infty} \exp \left(-\frac{t^{2}}{2}\right) d t\right)
$$

El principal objetivo de los distintos métodos de detección basados en energía consiste en la búsqueda de un umbral adecuado para la decisión. La elección de un umbral adecuado es importante para un buen desempeño del ED. Si el umbral es demasiado elevado, el SU puede decidir que hay espacio libre en el espectro cuando la señal de PU está presente, y su transmisión interferirá con la transmisión de PU, por el contrario, si el valor es demasiado bajo, el detector no reaccionará ante la ausencia de una señal en el canal y el SU perderá la oportunidad de utilizar el espectro. El umbral se obtiene mediante la ecuación 6 en donde $\boldsymbol{Q}^{\mathbf{- 1}}$ es la función inversa de $\boldsymbol{Q}$ evaluada en la Probabilidad de Falsa Alarma y equivale al número de muestras tomadas.

$$
\lambda=\sigma_{n}^{2}\left(Q^{-1}\left(P_{F A}\right) \sqrt{2 N}+N\right)
$$


La incertidumbre de piso de ruido se define como $\tau-1$, considerando este último, se reescribe la ecuación 7 referente al umbral en función a la probabilidad de falsa alarma, la misma se utiliza para el diseño del detector implementado.

$$
\lambda_{F A}=\tau \sigma_{n}^{2}\left[\frac{Q^{-1}\left(P_{F A}\right)}{\sqrt{\frac{N}{2}}}+1\right]
$$

\section{ENLACE DE COMUNICACIÓN BASADO EN RADIO COGNITIVO}

El presente trabajo propone la implementación de un enlace de comunicación basado en Radio Cognitiva que coloque en manifiesto la definición y principal característica del mismo. El enlace a implementar está constituido por el PU el cual transmite una frecuencia arbitraria y tiene prioridad en el uso de la banda. También se establece el respectivo Transmisor y Receptor Cognitivo perteneciente al SU, este deberá trasladarse a la banda de frecuencia libre más próxima en caso de que el PU ocupe la banda en que el SU está transmitiendo, sin que afecte ni interrumpa la comunicación entre Transmisor y Receptor del SU, la Fig. 3 muestra la arquitectura del sistema propuesto.

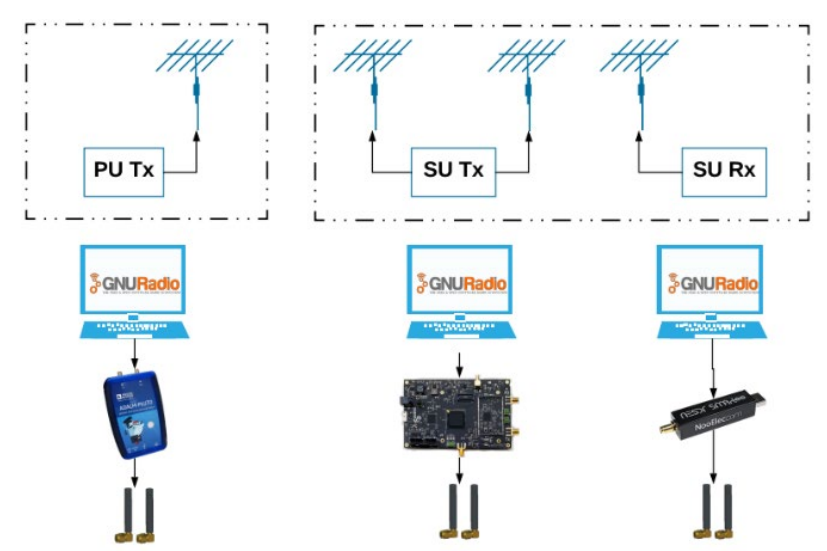

Fig. 3 Arquitectura del Sistema a Implementar

Existe en el mercado una gama extensa de dispositivos SDR. Para el presente trabajo se selecciona los dispositivos Blade RF x40, Pluto-SDR y RTL-SDR para su realización y posterior ejecución. Los dispositivos fueron elegidos conforme a su funcionalidad y características técnicas, las mismas que proveen los requerimientos necesarios para llevar a cabo la implementación del enlace.

Los SDR cumplen las funciones de RF e IF, mientras que GNU Radio realiza todas las funciones de banda base y reconfigura los SDR utilizados. GNU Radio controla las tarjetas Blade RF, RTL-SDR y PlutoSDR a través de OSMOCOM (gr-osmosdr) y IIO (gr-iio) respectivamente, estos controladores de hardware han sido creados específicamente para entablar la comunicación entre las tarjetas y el programa. La Fig. 4 muestra las funciones llevadas a cabo por GNU Radio en la cadena de Transmisión y Recepción por parte del SU.

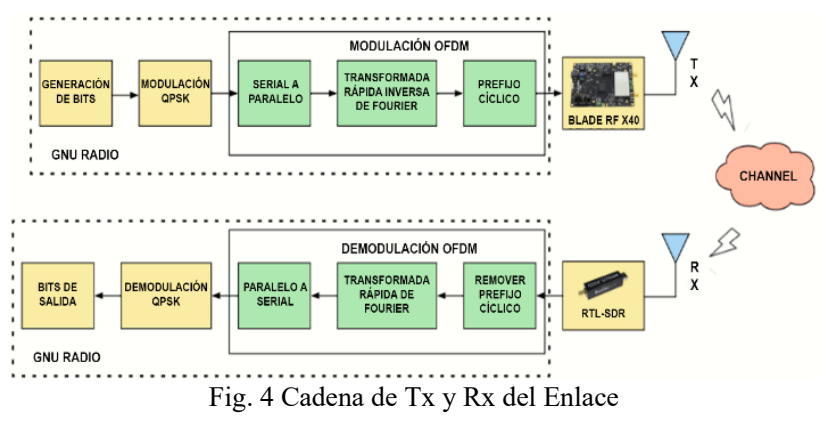

Es necesario tener en consideración ciertos parámetros para implementar el método de detección de espectro seleccionado, es el caso del Detector de Energía. Estos parámetros son la probabilidad de detección, falsa alarma e incertidumbre de ruido; por tal razón, los requerimientos son preestablecidos a base de la norma IEEE 802.22 y el estándar es el único en vigor referente a la tecnología RC. La TABLA I presenta el valor de los datos que se utilizan para la configuración del detector.

TABLA I

PARÁMETROS PARA CONFIGURACIÓN DEL DETECTOR DE ENERGÍA

\begin{tabular}{lc}
\hline \hline Parámetros de Configuración & Valores \\
\hline Probabilidad de Falsa Alarma $\left(\mathrm{P}_{\mathrm{fa}}\right)$ & $\leq 0.1(10 \%)$ \\
Probabilidad de Detección $\left(\mathrm{P}_{\mathrm{d}}\right)$ & $\leq 0.9(90 \%)$ \\
Incertidumbre de Ruido $(\tau)$ & 0.1 \\
Potencia de Ruido & $5 \times 10^{-9} \mathrm{~W}$ \\
Modulación & OFDM \\
\hline \hline
\end{tabular}

\section{A. Detector de Energía en GNU Radio}

Un nuevo bloque de ED se desarrolla e implementa en las bibliotecas de GNU Radio Companion [15]. Los parámetros de entrada del bloque ED son: el número de muestras, la varianza de ruido, la probabilidad de falsa alarma e incertidumbre de ruido. El ED desarrollado se prueba, como se muestra en el diagrama de bloques de la configuración experimental de la Fig. 5.

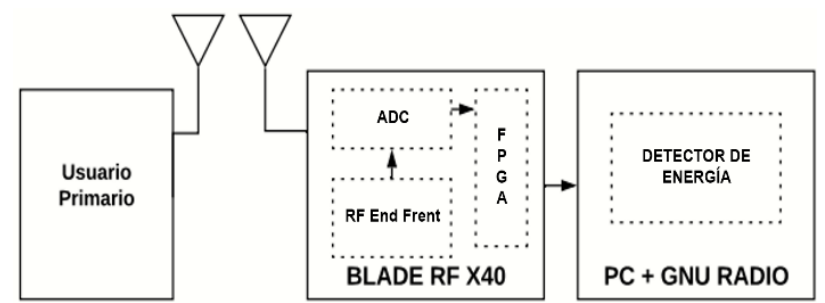

Fig. 5 Diagrama de bloques de la configuración experimental de detector de energía

\section{B. Detección y Transmisión del Transmisor Cognitivo}

La detección y la transmisión se implementan en paralelo en el transmisor cognitivo. Este transmisor realiza primero el sensado y a base de los resultados obtenidos toma las diferentes decisiones. La Fig. 6 ilustra de mejor manera lo anteriormente expuesto. Cabe destacar que mientras se realiza el sensado no desarrolla la trasmisión de datos, en caso de ejecutarse la transmisión se detiene hasta obtener el resultado del detector para proceder en la toma de decisiones. 
Una vez realizado el sensado existen dos posibles escenarios:

- Permanecer en la misma frecuencia, ya que esta se encuentra libre y no existe presencia de Usuario Primario o

- Trasladarse a la próxima frecuencia disponible puesto que ha detectado presencia de Usuario Primario y debe abandonarla inmediatamente sin interrumpir el enlace de comunicación.

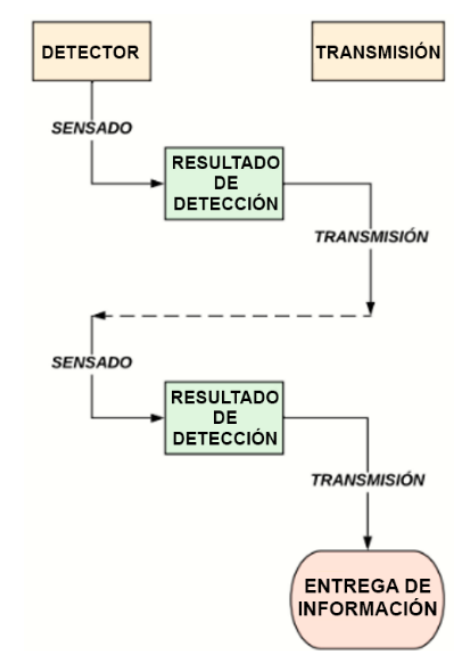

Fig. 6 Detección y Transmisión ejecutadas por el Transmisor Cognitivo

\section{RESULTADOS}

En la implementación del sistema CR, se usaron tres SDR, dos computadoras personales (PC) y una computadora portátil, como se muestra en la Fig. 7. La PC con SDR A actúa como transmisor de PU, la PC con SDR B actúa como Transmisor por parte de SU y la portátil con SDR 3 actúa como el receptor del SU.

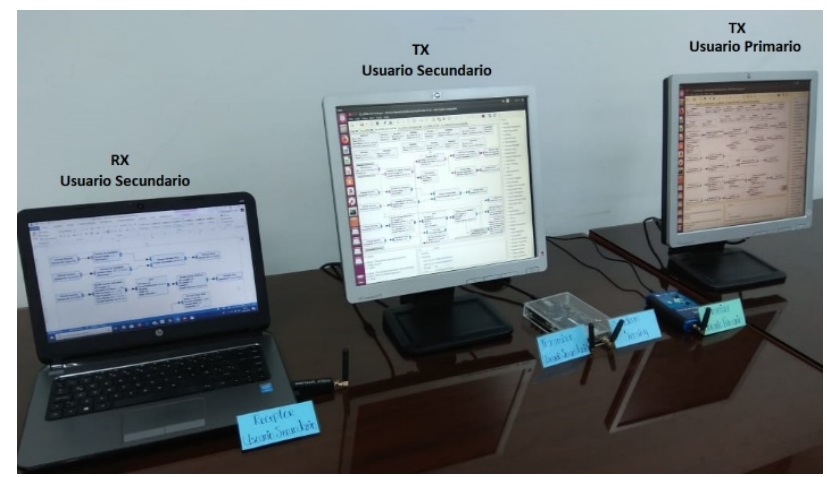

Fig. 7 Pruebas de la Arquitectura de GNU Radio

La frecuencia central de Transmisor PU se fija en $370 \mathrm{MHz}$ para evitar interferencias, cabe mencionar que con anterioridad se realiza un sensado del espectro en la ciudad de Loja, sitio de pruebas del enlace para determinar las frecuencias disponibles y las que poden ser seleccionadas para la implementación del presente trabajo.

Luego de finalizar la elaboración de los bloques necesarios para la implementación del Transmisor Cognitivo, se realiza la gráfica ROC, es decir, la curva de probabilidad de detección frente a la probabilidad de falsa alarma. Esta gráfica se usa a menudo para ilustrar y cuantificar la capacidad de detección del detector de energía, se ha creído conveniente realizarla para verificar el detector de energía implementado. La TABLA II muestra los parámetros utilizados en este documento para la evaluación del desempeño.

TABLA II

PARÁMETROS DE EVALUACIÓN DEL DESEMPEÑO DE DETECTOR DE ENERGÍA

\begin{tabular}{lc}
\hline \multicolumn{1}{c}{ Nombre } & Valor \\
\hline N. de Usuarios Primarios & 1 \\
Modulación de Usuario Primario & OFDM \\
N. de Usuarios Secundarios & 1 \\
Rango de SNR & $-21 \mathrm{~dB}$ \\
N. de muestras & 170000 \\
\hline \hline
\end{tabular}

La Fig. 8 muestra las curvas ROC experimentales obtenidas para el detector de energía, con diferentes valores de SNR.

Para obtener este gráfico, un SDR (Blade RF) sirve como detector y el otro (PlutoSDR) envia señales OFDM a diferentes amplitudes, es decir, varia el parámetro amplitud del transmisor. Las condiciones que se toman en consideración son las siguientes:

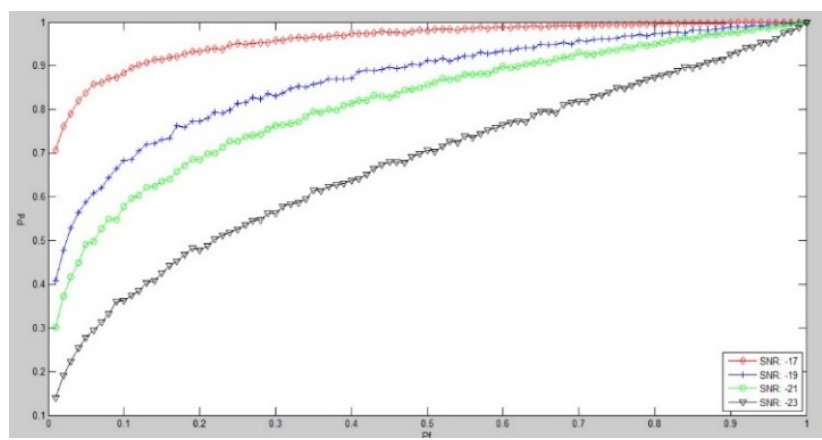

Fig. 8 Característica de funcionamiento del receptor para el detector de energía

- La detección de energía calculada es la de un canal con un ancho de banda de $1 \mathrm{MHz}$ con una frecuencia central igual a $400 \mathrm{MHz}$.

- Los SDRs se colocan en un ambiente interior (laboratorio), separados varios metros de distancia (entre 1 a 5 metros).

Como era de esperar, mientras más altas sean las muestras $(\mathrm{N})$, mejor será la calidad de la detección. El rendimiento del detector de energía no solo depende de la SNR, sino también del número de muestras utilizadas para la detección.

Una elevada probabilidad de falsas alarmas puede conducir a la pérdida de una potencial oportunidad de transmisión que los Usuarios Secundarios pueden aprovechar. Por otro lado, una detección perdida puede resultar en una interferencia catastrófica y perjudicial para los usuarios con licencia.

\section{A. Transmisor PU}

El flujo de datos desde su origen hasta el frente de RF se esquematiza en la Fig. 9.

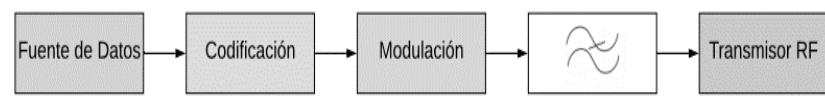

Fig. 9 Diagrama de bloques de TX de PU 
Se convierte el flujo de datos digitales en paquetes y se añade header/tag a dichos paquetes para su identificación. Posteriormente sigue la conversión de los datos en un esquema de constelación. A continuación, se asignan los puntos de constelación correspondientes. En esta etapa, los datos se representan como números complejos y están en serie, en este momento se pueden insertar símbolos piloto conocidos asignados con esquemas de mapeo también conocidos. Luego se aplica una conversión de serie a paralelo y se realiza la operación IFFT en los datos complejos paralelos. Los datos de salida de esta operación se agrupan nuevamente, según el número de subportadoras de transmisión requeridas. El prefijo cíclico se inserta en cada bloque de datos de acuerdo con la especificación del sistema y los datos se multiplexan de manera serial. En esta etapa, los datos están modulados por OFDM y listos para ser transmitidos. La tarjeta Pluto-SDR se utiliza para transformar los datos digitales en el dominio del tiempo, convertirlos a frecuencia de transmisión y enviarlos a través de un canal inalámbrico. La Fig. 10 indica la señal generada por el PU.

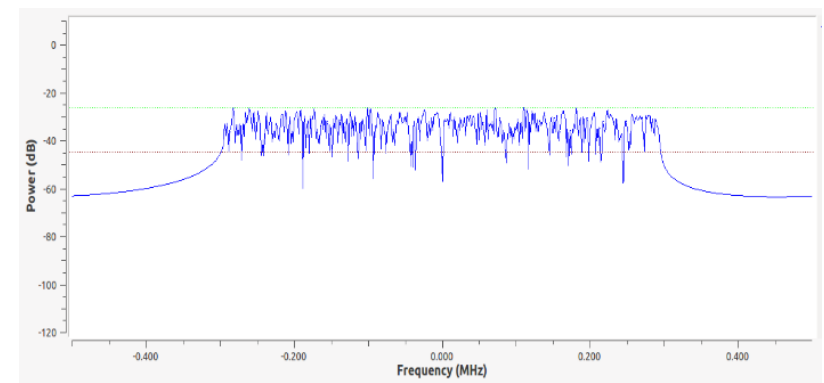

Fig. 10 Señal de Transmisión de PU generada en GNU Radio

\section{B. Transmisor $S U$}

El sistema RC consta de dos bloques de funcionamiento principales: detección de espectro y decisión del espectro. Posteriormente con la realización de dichos bloques se procede con la transmisión de datos. La detección de espectro se utiliza para sensar el espectro y detectar la presencia de la PU en el espectro escaneado. La decisión del espectro es responsable de identificar la frecuencia, establecer la sincronización y toma una decisión sobre cuándo y cómo comunicarse. La Fig. 11 ilustra la señal resultante del sistema CR generado.

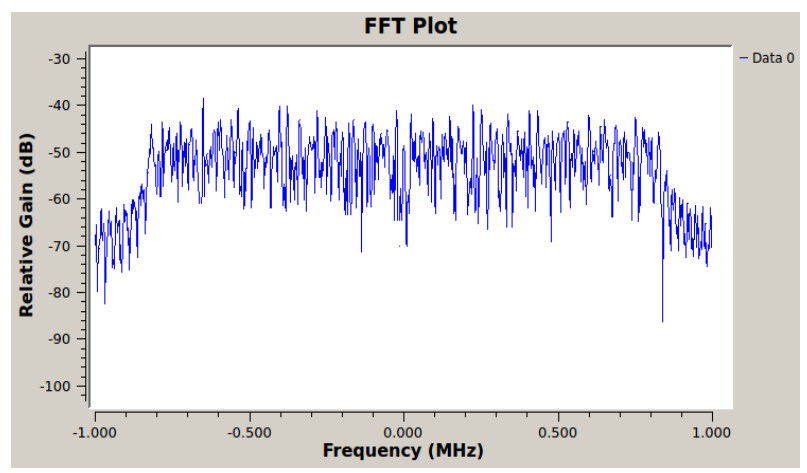

Fig. 11 Señal de Transmisión de SU generada en GNU Radio

\section{Receptor $S U$}

La forma de onda en el dominio del tiempo que se recibe por medio del RTL-SDR es detectada y posteriormente evaluada por los bloques creados anteriormente, la señal se dirige al bloque AGC para la corrección del nivel de potencia y luego al bloque de sincronización de tiempo que ejecuta la estimación del desplazamiento del tiempo del símbolo y la corrección del desplazamiento de la frecuencia de la portadora. El bloque Schmidl \& Cox OFDM synch realiza la estimación utilizando la auto correlación del preámbulo.

Posteriormente, la señal pasa por el encabezado y el flujo de carga útil que va al bloque FFT, luego el bloque FFT realiza la conversión de frecuencia y demodula las sub-partes individuales. La señal de dominio de frecuencia del encabezado y la carga útil pasa por la estimación de canal y el ecualizador de trama iguala el canal y corrige la frecuencia y la fase. Subsiguientemente los bloques del serializador OFDM eliminan los símbolos piloto, y pasan por el bloque Constellation Decoder el que se encarga de decodificar la carga útil para extraerla y re-empaquetarla, finalmente guarda la información receptada en un archivo para verificar la correcta llegada de los datos. La Fig. 12 muestra la señal resultante.

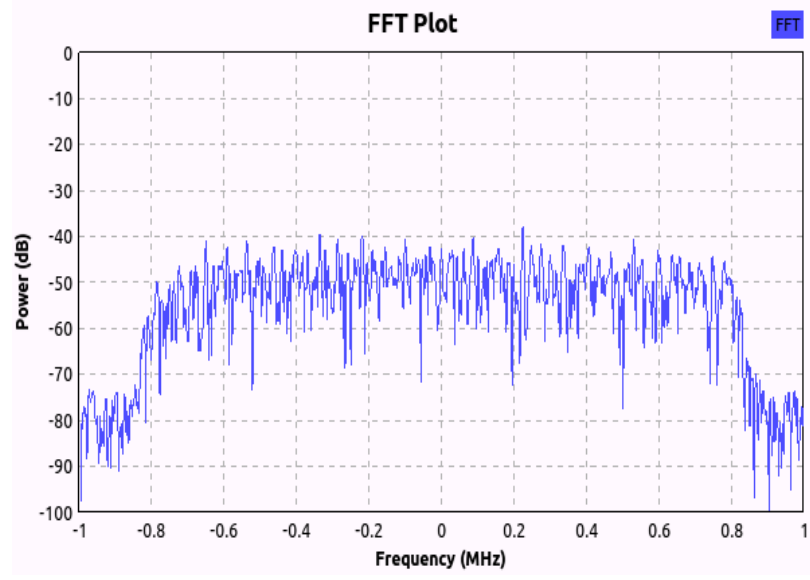

Fig. 12 Señal de Recepción de SU generada en GNU Radio

\section{DISCUSIÓN}

El estudio previo acerca de la tecnología de Radio Cognitiva y bibliografía referente a su implementación permite contrastar cabalmente los requerimientos necesarios y proporciona el principio básico para la selección de los elementos principales y fundamentales que conforman el enlace de comunicación.

El detector de Energía, seleccionado como método de detección para realizar el sensado de espectro, muestra un alto índice de respuestas positivas a la hora de detectar la presencia o no de Usuarios Primarios, es importarte resaltar que existen algunos falsos positivos, se deduce que dichos resultados se obtienen debido a la simplicidad de su arquitectura, se elabora la curva ROC para ilustrar y cuantificar la capacidad de detección del detector de energía, la Fig. 7 ilustra los resultados obtenidos.

Se puede contrastar que la herramienta de software GNU Radio seleccionada, presenta versatilidad y facilidad para la creación de los bloques requeridos. Es necesario un estudio previo de la funcionalidad del mismo y establecer un punto de partida para la creación y/o modificación de los bloques. El lenguaje de programación seleccionado fue 
$\mathrm{C}++$, el cual presenta resultados favorables y facilidad a la hora de adaptar las especificaciones necesarias.

Los resultados preliminares del trabajo experimental muestran que SDR proporciona flexibilidad en el diseño y comprobación de un sistema de comunicación de manera práctica, considerando las condiciones reales del canal. La eficiencia del enlace implementado es exitosa, al realizar la comparativa entre un enlace normal de comunicaciones y uno basado en sistemas de Radio Cognitiva se puede constatar las ventajas de la tecnología estudiada y la puesta en marcha de la misma que pone en manifiesto la definición y objetivo principal del tema de estudio de la presente investigación.

Este trabajo actualmente mantiene una limitante derivado del sistema radiante seleccionado el cuál presenta pérdidas y baja ganancia que lo hace vulnerable al enlace implementado frente al ruido y su distancia de propagación no es extensa.

\section{CONCLUSIONES}

La propuesta tecnológica presentada evidencia el funcionamiento adecuado del sistema y logra satisfacer el objetivo principal de implementar un enlace de comunicación basado en Radio Cognitiva y Radio Definido por Software.

La herramienta GNU Radio aporta considerablemente los procesos Cognitivos, se logra establecer el enlace planteado y se lleva a cabo las tareas de detector de espectro, detector de energía y decisión del espectro.

Se logra evidenciar que es factible el funcionamiento de un enlace cognitivo a través de herramientas de hardware y software, las mimas que no representan costes elevados y son de sencilla adquisición.

Los sistemas radiantes o grupo de antenas usados se establecen como un elemento primordial para el mejoramiento de las prestaciones técnicas de un enlace basado en los conceptos RC y expuestos de forma práctica sobre sistemas de radiocomunicaciones SDR.

\section{REFERENCIAS}

[1] K. Larson, L. Cacciatore, S. Crawford, T. Eng, E. Jacobs, J. Jackson, J. Levin, B. Luther, M. Marcus, T. Maguire, B. Nelson, S. Persaud, R. Repasi, B. Romano, T. Stanley, J. Williams, and J. Wong. (2002) "Spectrum policy task force," Spectrum policy task force. pp. $147-158$

[2] J. Mitola III. (2001). "Cognitive Radio for Flexible Mobile Multimedia Communications," Mobile Networks Applications., vol. 6 , no. 5, pp. 435-441

[3] J. Ma, Y. Li, and B. Hwang. (2009). "Signal Processing in Cognitive Radio," Proc. IEEE, vol. 97, no. 5, pp. 805-823.

[4] J. Banerjee, and K. Karmakar. (2012). "A Comparative Study on Cognitive Radio Implementation Issues"

[5] S. Shellhammer. (2008). "Spectrum Sensing in IEEE 802.22" Qualcomm Inc.

[6] F. Mejia, (2017). “Análisis de ocupación de canales para redes de Radio Cognitiva en el espectro de señales GSM durante un periodo de seis meses", Universidad Nacional de Colombia - Sede Bogotá

[7] Pedraza, L., Forero, F., y Páez, I. (2012) "Detección de espectro para radio cognitiva.” Ingeniare. Rev. Chil. Ing. vol. 20, no. 2, pp. 197-210.

[8] Z. Xuping, and P. Jianguo. (2007). "Energy-detection based spectrum sensing for cognitive radio." en IET Conference on Wireless, Mobile and Sensor Networks 2007 (CCWMSN07). vol. 2007, pp. 944-947.

[9] Z. Huang, W. Wang, and Y. Zhang. (2011). "Design and implementation of cognitive radio hardware platform based on
USRP." en IET International Conference on Communication Technology and Application (ICCTA 2011). pp. 160-164.

[10] S. Ricardo, F. Yoshimura, S. Mathilde, J. Dantas, A. Vicente, J. da Cruz Jr, J. Bazzo, and D. Melgarejo. (2018). "A USRP based scheme for cooperative sensing networks", $4^{\circ}$ Workshop de Redes de Acesso en Banda Ancha. Conferencia. XXXII Simpósio Brasileiro de Redes de Computadores e Sistemas Distribuídos

[11] FCC, "Notice of Proposed Rulemaking (NPRM 03 322): Facilitating Opportunities for Flexible, Efficient and Reliable Spectrum agile Radio Technologies."

[12] A. Mohammad. (2017). "Spectrum Access and Management for Cognitive Radio Networks". Springer

[13] P. Thakur, G. Singh, and S. Satasia. (2016). "Spectrum sharing in cognitive radio communication system using power constraints: A technical review", Perspectives in Science. Volumen 8. pp. 651653. https://doi.org/10.1016/i.pisc.2016.06.048

[14] R. Krishnan, R. Ganesh, S. Kaviya, N. Pragadeesh, C. Rahul, and S. Santhana. (2018). "Software defined radio (SDR) foundations, technology tradeoffs: A survey". IEEE Xplore. 10.1109/ICPCSI.2017.8392204

[15] R. Patil, D. Kulat, and A. Gandhi. (2018). "SDR Based Energy Detection Spectrum Sensing in Cognitive Radio for Real Time Video Transmission". Modelling and Simulation in Engineering. https://doi.org/10.1155/2018/2424305

[16] D. Teguig, M. Salah, and S. Sadoudi. (2020). "Analysis study and SDR implementation of GoF-based spectrum sensing for cognitive radio". IET Digital Library. vol. 14. pp. 857 - 864. 10.1049/ietcom.2019.0711

[17] D. Cabric, S. Mishra, and R. Brodersen. (2004). "Implementation issues in spectrum sensing for cognitive radios," in Conference Record of the Thirty-Eighth Asilomar Conference on Signals, Systems and Computers. vol. 1, pp. 772-776.

[18] D. Plata and A. Reátiga. (2012). "Evaluation of energy detection for spectrum sensing based on the dynamic selection of detectionthreshold," Procedia Eng. vol. 35. pp. 135-143

[19] Y. Liu, C. Zeng, H. Wang, and G. Wei. (2010). "Energy detection threshold optimization for cooperative spectrum sensing." 2nd International Conference on Advanced Computer Control. pp. $566-570$.

[20] V. Rodríguez., and J. Sánchez. (2014). "Empowering Software Radio: IT++ AS A GNU Radio Out-Of-Tree Implementation," IEEE Lat. Am. Trans. vol. 12, no. 2. pp. 269-276,

[21] J. Jussif, A. Arnez, L. da Silva, C. Rodriguez, C. Medina, and P. Gonzalez. (2018). "Real Time SDR Cognitive Radio System for Cooperative Spectrum Sensing in the $700 \mathrm{MHz}$ Brazilian Digital TV Band". IEEE-APS Topical Conference on Antennas and Propagation in Wireless Communications (APWC). IEEE Xplore. 10.1109/APWC.2018.8503674 